Chorologie, biologie, écologie et transmission des virus

\title{
De quelques biotopes larvaires de Culicoides Cératopogonidés étudiés en Alsace, dans les Vosges et en Lorraine
}

\author{
par J. CALLOT, M. KREMER et B. MOLET
}

Institut de Parasitologie, Faculté de Médecine, 3, rue Kaberlé, F 67000 Strasbourg

\section{Résumé.} trées.

Remarques sur divers biotopes larvaires de Cératopogonidés et sur les espèces rencon-

\section{Summary.}

Larval biotops of Culicoides in Alsace, Vosges and Lorraine (France).

Notes on some larval biotops of Ceratopogonides and on found species.

Depuis de nombreuses années, nous récoltons des échantillons de boues dans des endroits susceptibles d'être des gîtes larvaires de Culicoides. Ces prélèvements sont rapportés au laboratoire, mis dans des bocaux recouverts d'une plaque de verre et les adultes qui en éclosent sont capturés et montés en vue de leur détermination. Nous rapportons ici quelques particularités des différents gîtes ainsi explorés et indiquons les conditions propices au développement de formes larvaires, en particulier dans les Vosges et en Alsace. 
Nous avons prélevé les bords de mares, d'étangs, les berges de ruisseaux et de sources et le sol de marécages et de tourbières. Nous nous sommes intéressés aux collections d'eau temporaires telles qu'ornières, flaques, ainsi qu'à du matériel végétal en décomposition: souches pourries, crottins ou bouses de vache, champignons en décomposition. Enfin, à une sorte particulière de gîtes représentée par les trous d'arbres.

Un certain nombre de ces prélèvements n'ont donné lieu à aucune éclosion, ce sont ceux présentant un matériel trop sec. De même, les champignons se sont toujours révélés négatifs.

Le crottin et les bouses ont donné éclosion à $C$. chiopterus et $C$. dewulfi.

Le $\mathrm{pH}$ de nombreux gîtes a été mesuré et il peut varier de moins de 6 pour le sol de tourbières, à 7 pour la majorité des prélèvements constitués surtout de terre, et peut s'élever jusqu'à 8,2 pour le contenu des trous d'arbres.

Certains éléments chimiques ont été mesurés, en particulier, le $\mathrm{NaCl}$ qui peut être très variable. On connaît un certain nombre d'espèces halophiles tels $C$. halophilus, C. salinarius, C. circumscriptus, C. nubeculosus, C. riethi rencontrés en Lorraine et en Alsace. $\mathrm{Si}$ on étudie le type de gîtes dont sont issus les espèces de Culicoïles, on constate que certaines n'ont pas de préférences particulières quant à la nature du sol où elles se développent, par exemple, $C$. odibilis qui éclot de gîtes extrêmement variés tels que tourbières, mares de forêts, berge d'étang et $C$. fascipennis sortant de la boue neutre de mares, de ruisseaux très riches en $\mathrm{NaCl}$ des régions des mines de potasse, de sol acide des tourbières.

Par contre, d'autres espèces sortent de gîtes bien précis tel $C$. impunctatus qui n'est trouvé que dans le sol acide des tourbières.

Les trous d'arbres représentent un biotope très particulier et certaines espèces de Culicoïdes leur sont inféodées, en particulier, C. sylvarum, C. fagineus, C. semimaculatus et, quelquefois, $C$. obsoletus.

Nous avons prélevé dans des trous de hêtres, de sapins et d'érables. Curieusement, toutes les espèces écloses de trous d'arbres de nos régions ont les ailes táchetées.

Les Cardaires (Dipsacus silvestris) représentent un gîte particulier pour certaines espèces de Dasyhelea, mais aucun Culicoides n'en est éclos jusqu'ici.

Pour terminer ces brèves remarques, indiquons notre ignorance quasi totale des gîtes d'espèces de Culicoides pourtant fréquemment rencontrées dans notre région: $C$. obsoletus, $C$. chaetophtalmus et $C$. riouxi. Ces espèces habitent-elles des gîtes que nous n'avons pas su reconnaître ou bien nos méthodes d'élevage en laboratoires ne permettent-elles pas de les faire éclore ? Ceci prouve que si des progrès ont été réalisés dans la connaissance des biotopes larvaires, de nombreuses recherches restent encore à faire pour mieux comprendre la biologie des Cératopogonidés. 